\title{
Decision time, subjective probability, and task difficulty
}

\author{
GEORGE WRIGH'T \\ Bristol Polytechnic, Bristol, England \\ and \\ PETER AYTON \\ City of London Polytechnic, London, England
}

\begin{abstract}
This study analyzed the relationships between decision time, subjective probability, and task difficulty in the context of a probability assessment task involving memory search. The results indicate that decision time and subjective probability do not yield identical functions. Also, decision time increases as subjective task difficulty increases. A similar relationship obtains between decision time and a measure of objective task difficulty. These latter two findings are inconsistent with Hogarth's (1975) prediction of a nonmonotonic relationship between decision time and task difficulty.
\end{abstract}

Subjective probabilities are the prime numerical inputs to decision analysis (see Raiffa, 1968), cross-impact analysis (Dalkey, 1972), fault-tree analysis (Fischhoff, Slovic, \& Lichtenstein, 1978), and many other management technologies. Often actuarial or relative-frequency-based data are unavailable or believed to be unreliable as direct inputs. In such cases, probability assessments must be provided by judgment. Two commonly used direct methods for probability assessment are point estimates and odds estimates. In decision analysis, a commonly used indirect method is the reference wager technique. Imagine this event: last year's winner of the New York marathon may win again this year. Wager $A$ specifies that if the same person wins, you will win $\$ 10,000$; otherwise, you win nothing. Under Wager $B$, you win $\$ 10,000$ with probability $p$, and nothing with probability $1-p$. Wager B may be played by blindly drawing a ball from an urn filled with identically shaped balls, a proportion $p$ of which are white, and $1-p$ are black; you win if a white ball is drawn. There is one restriction on Wager B: the ball will not be drawn until the day of the New York marathon. Thus, under either wager, the consequences for you are identical: you may or may not win $\$ 10,000$ on the day of the New York marathon.

Depending on whether you prefer Wager A or B, the probability $p$ is varied until you are indifferent between the two wagers. The indifference probability associated with Wager B gives an indirect measurement of the subjective probability for the event in question. (See Wright,

This research was written up with the aid of a British Economic and Social Research Council project grant (C00232037) awarded to the first author. We wish to thank Alastair McClelland for invaluable statistical advice and much ungrudgingly suffered computational labor. Address correspondence to George Wright, Bristol Business School, Bristol Polytechnic, Coldharbour Lane, Frenchay, Bristol BS16 1QY, England.
1984, for a fuller discussion of probability assessment methods.)

Which of these three methods is the best for the elicitation of subjective probability? The empirical evidence is contradictory. Sometimes the indirect methods are inconsistent with the direct methods, and sometimes they are not. Beach and Phillips (1967) demonstrated consistency between probability estimates inferred from wagers and direct estimates. However, Winkler (1967) showed that statistically naive subjects were inconsistent between direct and indirect methods, whereas statisticians were not. Generally, direct odds estimates, perhaps because they have no upper or lower limit, tend to be more extreme than direct probability estimates. If subjective probability estimates derived by different methods for the same event are inconsistent, which method should be taken as the true index of degree of belief? In the present study we explored the relationships between a further indirect measurement of degree of belief, decision time, and direct subjective probability assessments.

In an early study, Cohen, Hansel, and Walker (1960) investigated the relationship between decision time and measures of subjective probability. Decision time was defined as the time taken to decide whether a ball could or could not be successfully rolled through a gap that varied in width. Subjective probability can be defined as degree of belief in the truth of a proposition, in this case the binary yes or no decision. Cohen et al. related binary decision times to corresponding subjective probabilities. They found that when subjective probability increased from 0 to 0.5 , implying increasing uncertainty between alternatives, decision time increased, whereas when subjective probability increased from 0.5 to 1.0 , implying decreasing uncertainty, decision time decreased. Using a task similar to that of Cohen et al., Branthwaite (1974) obtained inverted- $V$ relationships between decision time and 
two measures of subjective probability: confidence ratings on a $0 \%$ to $100 \%$ scale and estimates of number of successes from 10 trials. Branthwaite concluded that although decision time can be taken as an index of subjective probability, its interpretation requires calibration against another measure.

This relationship between decision time and confidence is not found for all tasks, however. For example, Ekehammar and Magnusson (1973) found that the length of time required by interviewers to make personality ratings of interviewees on a 5-point scale, after observing an interview, was a monotonic increasing function of the interviewers' degree of confidence in their ratings. Diener and Thompson (1986) observed an inverted-V function between confidence and decision speed when they asked subjects to judge whether a series of coin tosses were produced by a coin biased to produce more heads than tails. However, when an independent group of subjects was asked to judge whether the same sequences were produced by a fair coin, the responses did not follow an inverted-V. Although, as in the biased coin condition, decision times increased between $0 \%$ and $50 \%$ confidence levels, decision times above $50 \%$ (indicating increasing confidence that the series are random) remained relatively flat; there was no significant correlation between decision time and confidence above the $50 \%$ confidence level.

Geller and Pitz (1968) examined the relationship between confidence and decision speed in the revision of opinion. They concluded that decision speed may be a better index of the objective probabilities given by Bayes's theorem than are confidence ratings, because the relative changes in decision speed reflected the probabilities calculated by Bayes's theorem more closely than did confidence ratings. They argued that subjects may be unwilling to reduce their explicit confidence ratings after disconfirming information.

Independently, Phillips and Wright (1977) proposed a three-stage sequential model of the cognitive processes involved in answering a question. This model was developed in the context of probability responses given to general-knowledge questions. According to the model the cognitive processes involved follow up to three stages: a certainty response (i.e., either a "yes" or "no" answer or a probability estimate of 0 or 1 ) if the processes stop at Stage 1; a response consequent on a refusal to respond probabilistically (i.e., either a "don't know" answer or a .5 probability estimate) if they stop at Stage 2; and a truly probabilistic response (i.e., a probability estimate other than $0,0.5$, or 1 or a corresponding verbal expression) at Stage 3. Wright (1982) used this model to describe task influences on answers given to questions. Phillips and Wright's model implies that .5 probability responses are quicker than any probability response other than 0 or 1 . This prediction is in contradiction to the findings reviewed above.

Hogarth (1975) presented a model of a nonmonotonic relationship between decision time and task complexity, or difficulty, in the context of choices among multiattributed alternatives. In terms of this model, decision times for very simple tasks that require little information processing are short. Decision times are also short for very difficult or complex tasks for which the decision maker, due to the limits of information processing capacity, abbreviates task processing. According to Hogarth, decision time is longest when the decision maker's maximum information processing ability is reached.

Hogarth (1975) speculated that experts would have shorter decision times than novices because experienced decision makers may find familiar tasks easier. Hogarth also argued that if feelings of certainty are positively correlated with expertise, then his model may account for the short decision times previously found for certainty responses.

In this study, we investigated the relationship between decision times and subjective probability estimates in the context of probability assessments given to generalknowledge questions. In addition, two measures of difficulty were taken, one objective and one subjective, and the relationship of decision time to these was analyzed.

\section{METHOD}

A total of 37 British students attending Huddersfield Polytechnic voluntarily completed the Probability Assessment Questionnaire (PAQ; detailed in Phillips \& Wright, 1977), which contains 75 general-knowledge questions, such as "Which is larger? (a) Black Sea, (b) Caspian Sea." The full set of 75 questions and their two response alternatives are included in the Appendix of this paper. Since we had no a priori hypotheses concerning possible sex differences, this was not tested. The questions were sequentially presented by computer on a television screen. All parts of an individual question appeared simultaneously on the screen. The questions used were all roughly equal in length. Mean question length was 9.32 words, with a standard deviation of 2.52 words.

Each subject read detailed instructions concerning the task and ordering of responses before responding to $10 \mathrm{warm}$-up and familiarization questions that preceded the PAQ. The ordering of questions within the PAQ was randomized for each subject. To be presented with a question, the respondent had to hold down a release button for $3 \mathrm{sec}$, after which time the question appeared on the screen. The respondent was required to press either an " $a$ " or a " $b$ " button to indicate the correct answer. The time elapsed between the presentation of the question and the " $a$ " or " $b$ " response, which we call binary decision time, was recorded. The respondent was then required to indicate how sure he or she was of the answer by pressing one of seven buttons, which were labeled; $50 \%$ sure, $51 \%-59 \%$ sure, $60 \%-69 \%$ sure, $70 \%-79 \%$ sure, $80 \%-89 \%$ sure, $90 \%-99 \%$ sure, and $100 \%$ sure. The members of each set of response buttons were equidistant from the release button. Figure 1 shows the layout of response buttons. The detailed typewritten instructions that explained the task clarified use of the scale for percentage of confidence:

It extends from 50 to 100 , with 100 meaning absolutely sure to 50 meaning completely unsure. The two extreme buttons in the semicircle of buttons correspond to these assessments. Note that even if you had absolutely no idea what the correct answer was, you would still have a $50 \%$ chance of being correct as there are only two alternatives. For most of the questions, your 

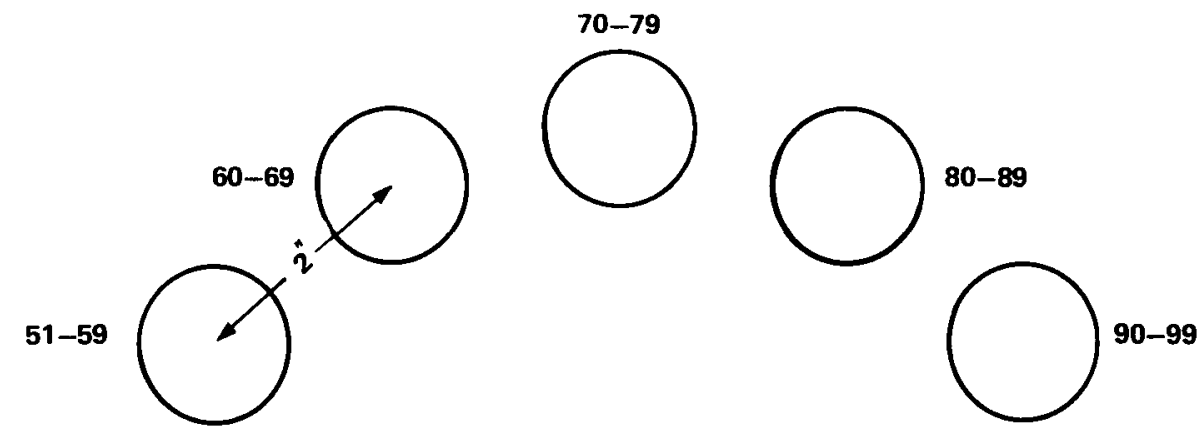

50
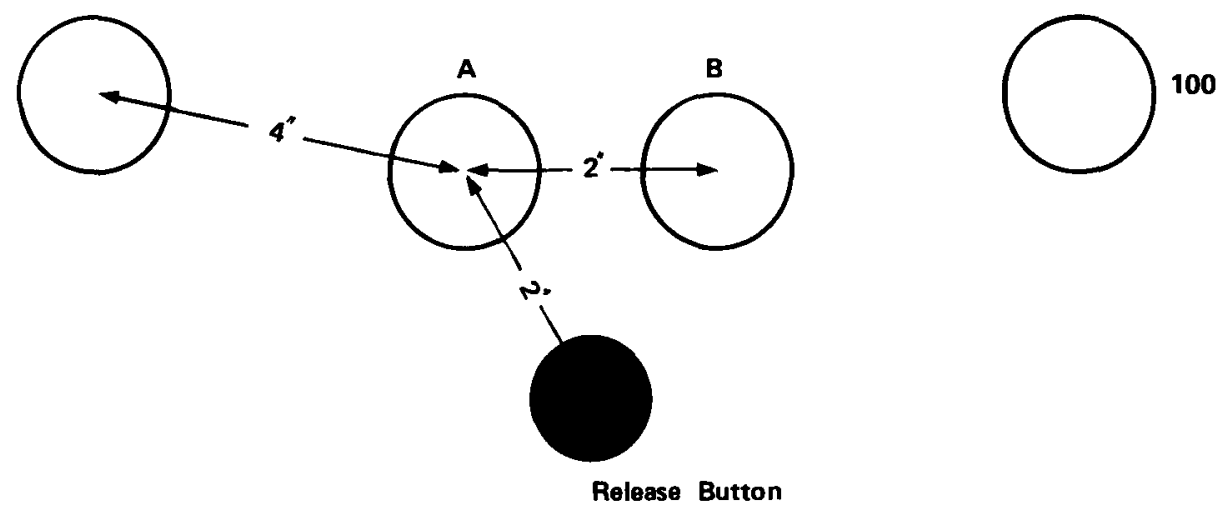

Figure 1. Layout of response buttons.

"\% sure" will probably lie between the extremes; that is you won't be absolutely sure, nor will you be completely unsure. You would therefore select a button between $50 \%$ and $100 \%$ to indicate how sure you were.

This rating scale was used because it seemed to be more natural for college students to express their confidence on a $50 \%$ to $100 \%$ scale than as a probability on a .5 to 1 scale.

The elapsed time between pressing either the " $a$ " or the " $b$ " button and pressing a confidence button, which we call probability estimation time, was also recorded.

Alternatives " $a$ " and ' $b$ " were each correct about $50 \%$ of the time. With this manipulation, we anticipated that any differences in the time for the respondent's finger to travel from either decision button to the confidence buttons would be counterbalanced.

After a confidence button had been pressed, the question disappeared from the screen and the respondent was required to indicate, on a 7-point scale ranging from extremely easy (1) to extremely difficult (7), how difficult he or she had found the question to answer. In this way an individual's subjective difficulty was measured for each question. When the scale had been marked, the subject was required to hold down the release button to signal the next question.

Objective difficulty was measured as overall proportion correct. This measure of task difficulty has been extensively used by Lichtenstein and Fischhoff (1977) and was computed for each question across the 37 subjects. The mean probability response given by a subject and his/her total number of $100 \%$ and $50 \%$ responses were also computed.

\section{RESULTS AND DISCUSSION}

\section{Subjective Probability and Decision Time}

From the literature it is unclear whether time to a binary decision or time to a probability estimate is the most reliable index of subjective probability. Branthwaite (1974) measured time to a binary decision-whether or not the subject thought a ball could be rolled through a gap-and then asked for an untimed confidence rating. Geller and Pitz (1968) utilized a similar routine in a "bookbag and poker chip" probability revision task. However, Cohen et al. (1960), using two groups of subjects, measured time to a binary decision with one group and time to a probability estimate with the other. They found that the time to make a binary yes or no decision was always shorter than the time to make a probability estimate at corresponding levels of subjective probability. Although an inverted-V relationship was obtained between binary decision time and subjective probability, no clear relationship was found between time to make a probability estimate and the estimate itself. For these reasons, our analysis focused on binary decision time rather than on total decision time.

Table 1 gives the means and standard deviations of the binary decision times for the $50 \%-100 \%$ range of subjective probability used in this study. A one-way analysis of variance (ANOVA) revealed that binary decision time was not constant across confidence levels $\left[F(6,240)^{1}=\right.$

Table 1

The Relationship Between Subjective Probability and Binary Decision Time

\begin{tabular}{crrrrrrr}
\hline & \multicolumn{6}{c}{ Probability Response } \\
\cline { 2 - 8 } Becison Time & \multicolumn{1}{c}{50} & $51-59$ & $60-69$ & $70-79$ & $80-89$ & $90-99$ & 100 \\
\hline$M$ & 11.74 & 12.55 & 12.46 & 11.32 & 9.83 & 9.93 & 7.46 \\
$S D$ & 3.83 & 5.36 & 5.00 & 4.50 & 3.06 & 4.39 & 2.69 \\
\hline
\end{tabular}


6.58, $p<.001]$. A linear trend test was significant $[F(1,240)=30.29, p<.001]$, as was a quadratic trend test $[F(1,240)=6.41, p<.01]$. By inspection it would appear that the presence of nonlinearity in the data may largely be attributable to the response times for $50 \%$ judgments, which were discrepantly low. A post hoc trend analysis (Gaito, 1965; Kirk, 1982) omitting the 50\% judgments confirmed this view; the linear component was again significant $[F(1,240)=33.61, p<.05]$, although there was no longer evidence for a quadratic trend $[F(1,240)=1.10, p>.05]$. Unplanned comparisons (Scheffé, 1953) showed that the 50\% response times were not significantly shorter than those for the next highest subjective probability category, $51 \%-60 \%[F(1,240)=$ $0.64, p>.05]$. Unplanned comparisons also showed that the binary decision times of $50 \%$ responses were similar to the other responses, omitting $100 \%$ and then taken as a group $[F(1,240)=0.57, p>.05]$, whereas the binary decision times of the $100 \%$ responses were shorter than the other responses, taken as a group $[F(1,240)=25.45$, $p<.05]$. The binary decision times for the $100 \%$ responses were also shorter than those for $50 \%$ responses $[F(1,240)=19.05, p<.05]$.

These results were inconsistent with Phillips and Wright's (1977) sequential model of probabilistic processing, in that the decision time of a $50 \%$ response was no shorter than that of other probabilistic responses, excluding $100 \%$ responses. There was also only moderate support for a strict monotonic decreasing relationship between binary decision time and associated confidence or probability over the half $(50 \%-100 \%)$ range of probability. The only unambiguous finding was that $100 \%$ responses showed a faster binary decision time than all other probabilistic responses.

Supporting this interpretation, the correlation between mean probability response and mean binary decision time across all subjects was found to be near zero $(r=-.002$, $p>.05$ ). However, on analyzing subjects as individuals, 13 gave a significant, negative intercorrelation between binary decision time and probability response, whereas the remaining 24 subjects gave near-zero correlations between these two variables. These results suggest the intriguing possibility that a monotonic decreasing relationship between binary decision time and confidence holds true for some people, whereas no relationship holds for others. Mean comparisons on the measure of reaction time between these two groups of subjects revealed no differences. These results should, of course, be treated with caution due to the small sizes of the subdivided sample.

We also considered analyzing in detail the time elapsed between the presentation of a question and the pressing of a confidence button, which we call total decision time. Although a one-way ANOVA revealed that total decison time was not constant across confidence levels $[F(6,240)$ $=4.59, p<.01]$, the form of the relationship shown in Figure 2 reveals that, as Branthwaite (1974) and Cohen et al. (1960) suggested, binary decision time is the major determinant of variations in total decision time.

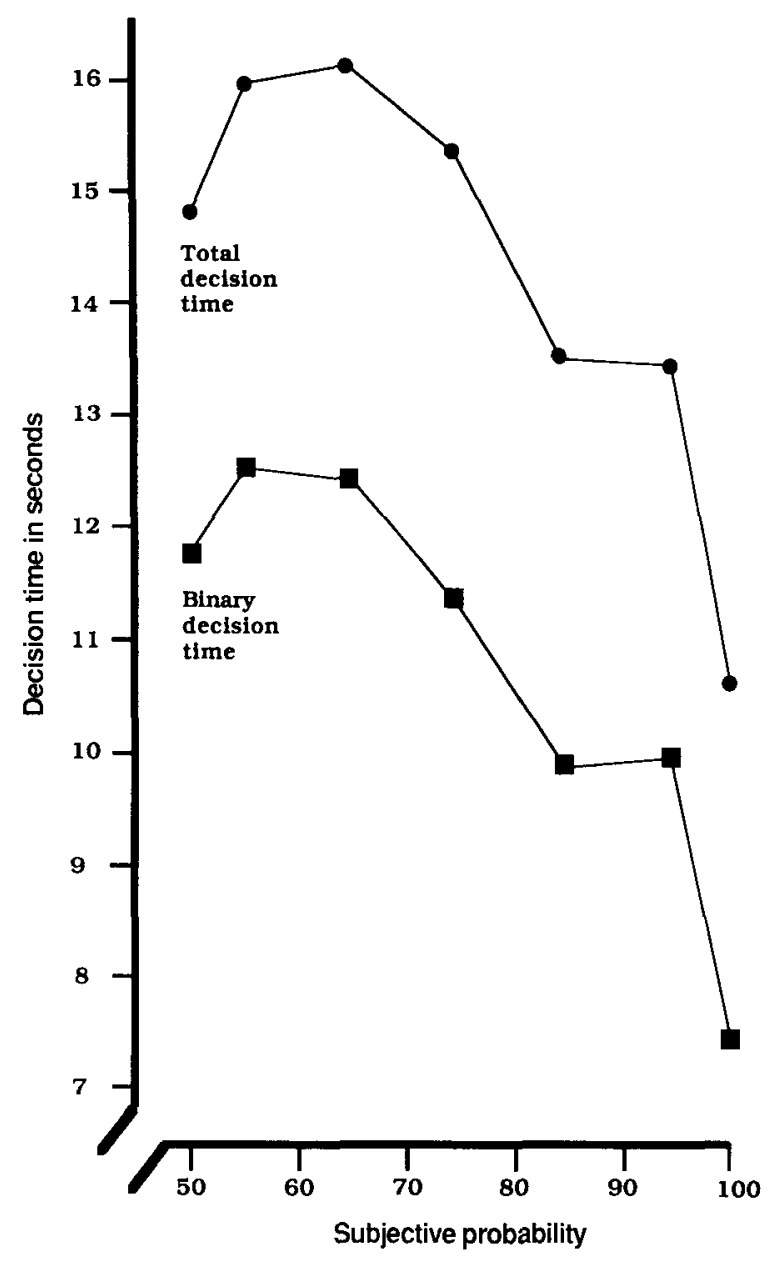

Figure 2. Relationship between subjective probability and decision times.

A one-way ANOVA revealed no significant relationship between probability estimation time and subjective probability $[F(6,240)=.78, p>.05]$. Trend tests were not significant. Mean probability estimation time was $3.52 \mathrm{sec}$, with a standard deviation of $2.4 \mathrm{sec}$. These findings imply that either (1) probability estimation involves an equivalent amount of probabilistic processing for each probability response range, or (2) probability estimation time involves only a simple mapping of subjective uncertainty onto a response scale, whereas probabilistic processing occurs in binary decision time, as Branthwaite (1974) and Geller and Pitz (1968) assumed. To generalize, if probabilistic processing does occur in binary decision time, this time index is not a clear-cut substitute for subsequently assessed probability across the half-range of probability utilized in the present experiment.

Why is there a difference between the present results and results of earlier research? The difference may be due to the nature of the tasks used. Cohen et al. (1960) and Branthwaite (1974) used a task that involved subjects' making decisions about rolling a ball through a gap that was varied in width. Clearly, the type of information processed by a subject varied little from decision to deci- 
sion. Similarly, Geller and Pitz (1968) used a probability revision task in which subjects were shown a sequence of red and white poker chips drawn from one of two bags containing either predominantly red or predominantly white poker chips. The subject's task was to decide, after each draw, which of the two bags was the data generator. In this paradigm, also, the task parameters remained very similar from decision to decision. Once a task is understood by a subject, it may be that binary time is a fairly pure indication of probabilistic processing involved in repetitive decisions.

By contrast, the probability assessment task used in the present study consisted of 75 questions, each entirely distinct from every other. The only similarity between questions was that each had only two possible answers, one of which was correct. Every question, by its nature, involved long-term memory search, which sometimes might have resulted in a great deal of question-relevant information being recalled, sometimes not. This information then had to be evaluated. Glucksberg and McCloskey (1981) reported evidence that suggests that subjects can make a "don't know" response faster than a true/false judgment when a search of memory reveals no relevant information. This is because, under such circumstances, there is no time-consuming evaluation of evidence. In the present study, the $50 \%$ responses plausibly consisted of a proportion of occasions when no evidence was obtained by subjects to delay an arbitrary choice; on other occasions, evidence might have been retrieved, but evaluation revealed it to be equivocal. Such a mixture may explain why the $50 \%$ responses were faster than allowable by a model predicting a linear relationship between subjective confidence and decision time (Branthwaite, 1974; Cohen et al., 1960) but not fast enough to fit the threestage model proposed by Phillips and Wright (1977). However, we found no evidence of a bimodal distribution of the binary decision times that resulted in $50 \%$ responses. In the present task, average binary decision time was about $11 \mathrm{sec}$; in Cohen's task, it was about $2.5 \mathrm{sec}$. In Geller and Pitz's (1968) task the average decision time was not reported, but perhaps we can assume that it was short since "decision times were recorded to the nearest millisecond" (p. 193).

If we accept that most real-life decisions are not made repetitively in static environments, we can conclude that in many situations decision time may lack reliability as an indicator of subjective probability.

\section{Subjective Difficulty and Decision Time}

Since Hogarth (1975) predicted a curvilinear relationship between task difficulty and decision time, mean decision times for each of the seven levels of subjective difficulty were calculated. Table 2 sets out the means and standard deviations. The mean of subjective difficulty was 3.84 , with a standard deviation of 1.12 .

A one-way ANOVA was significant $[F(6,233)=5.61$, $p<.05$ ], suggesting that binary decision time is related to subjective difficulty. Supporting Hogarth's (1975)
Table 2

The Relationship Between Subjective Task Difficulty and Binary Decision Time

\begin{tabular}{cccrrrrrr}
\hline & \multicolumn{7}{c}{ Subjective Task Difficulty } \\
\cline { 2 - 8 } Decision Time & 1 & \multicolumn{1}{c}{$\mathbf{2}$} & \multicolumn{1}{c}{ - } & \multicolumn{1}{c}{5} & \multicolumn{1}{c}{6} & \multicolumn{1}{c}{7} \\
\hline$M$ & 7.98 & 8.62 & 10.31 & 10.99 & 12.04 & 11.92 & 12.30 \\
$S D$ & 3.47 & 3.55 & 3.36 & 4.27 & 5.11 & 4.71 & 4.51 \\
\hline
\end{tabular}

model, a significant quadratic trend related binary decision time to subjective difficulty $[F(1,233)=4.25$, $p<.05$ ], but the observed function is clearly not of the form postulated by Hogarth (see Figure 3); with one slight exception, decision time increased with subjective difficulty and the items rated most difficult resulted in the largest decision times. Moreover, there was a significant linear trend $[F(1,233)=37.7, p<.005]$. This result suggests that as subjective difficulty increased, binary decision time increased.

A one-way ANOVA on the probability estimation times for the seven levels of subjective difficulty was not significant $[F(6,233)=1.3, p>.05]$.

Thus the obtained curvilinear relationship between decision time and subjective difficulty was not as pronounced as our interpretation of Hogarth's (1975) model would predict. The average number of $50 \%$ responses and $100 \%$ responses given by subjects were 13 and 17 , respectively, so it is assumed that the 7-point subjective difficulty scale utilized in the present study covered the very simple to complex range of task difficulty necessary for the valid application of Hogarth's model.

\section{Objective Difficulty and Decision Time}

To achieve a somewhat comparable analysis of the relationship between overall proportion correct (OPC) and decision time, the 75 items in the probability assessment questionnaire were divided into seven groups of 10 items differing in OPC. Five items were discarded from the analysis so that differences in OPC between the seven groups of items were maximized. Table 3 sets out the

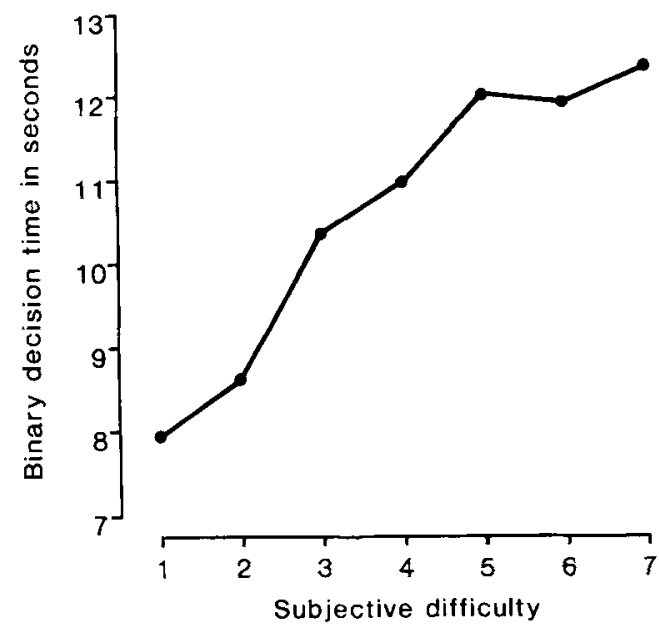

Figure 3. Relationship between subjective difficulty and binary decision times. 
Table 3

The Relationship Between Objective Task Difficulty and Binary Decision Making

\begin{tabular}{cccccccc}
\hline \multirow{2}{*}{$\begin{array}{c}\text { Binary } \\
\text { Decision Time }\end{array}$} & 0.87 & 0.80 & 0.74 & 0.70 & 0.65 & 0.51 & 0.41 \\
\hline$M$ & 8.93 & 9.64 & 10.01 & 9.85 & 12.73 & 10.65 & 11.07 \\
$S D$ & 6.71 & 6.76 & 6.20 & 6.51 & 8.94 & 6.12 & 6.74 \\
\hline
\end{tabular}

means and standard deviations of the binary decision times associated with the seven levels of OPC. Mean OPC was 0.63 , with a standard deviation of 0.7 .

A one-way ANOVA revealed that binary decision time was not constant across OPC levels $[F(6,252)=11.75$, $p<.002]$. In support of Hogarth's model, a significant quadratic trend related binary decision time to OPC $[F(1,252)=5.39, p<.05]$, but there was also a significant linear trend $[F(1,252)=34.47, p<.002]$. This result is analogous to that obtained with subjective task difficulty. Inspection of the shape of the function (see Figure 4) reveals that although it bears some resemblance to what Hogarth's model predicts, there are also discrepancies. Thus, there was an initial increase in decision times with increasing objective difficulty (decreasing OPC) but there were subsequently two separate downward turns, and the most difficult questions (lowest OPC) had the second highest decision times.

A one-way ANOVA revealed no evidence of a significant relationship between probability estimation time and OPC.

Although there was some evidence of a curvilinear relationship between binary decision time and both subjective and objective task difficulty, in each case the relationship was not of the type postulated by Hogarth (1975). Furthermore, in each case there was strong evidence of a linear relationship. As subjectively perceived task difficulty increased, binary decision time increased. Were subjective and objective task difficulties measuring the same thing? To answer this question, the mean subjective rating was correlated across the 75 items with the percentage of subjects choosing the correct alternative. The

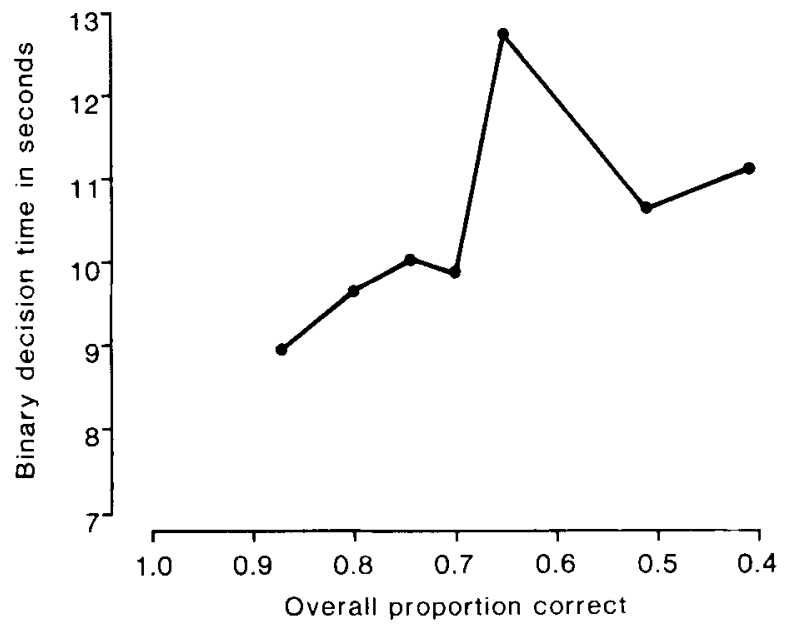

Figure 4. Relationship between objective difficulty and binary decision times. obtained correlation was -0.49 , suggesting that the two measures shared some variance in common.

\section{CONCLUSION}

In a probability assessment task using generalknowledge questions, decision time and associated degree of belief were not interchangeable. However, it must be noted that the present dual-task test may not have been fair to either of the two reaction time measures (binary decision, probability estimation) because of the fact that the two tasks were concatenated (binary decision, followed by probability estimation). The earlier binary decision may have affected the later probability estimation reaction time, for example, by letting its processing occur concurrently or by fatiguing the subject. Conversely, the later probability estimation may have affected the earlier binary decision reaction time, for example, by competition for resources during concurrent processing. It may be that if each task were run alone with a separate group of subjects, both tasks, not only the binary decision task, would show comparable effects of subjective probability and subjective and objective task difficulty.

Nevertheless, in the present study the only clear-cut finding was that $100 \%$ assessments show a faster binary decision time than all other assessments. The task of assigning probabilities to factual matters requires long-term memory search, perhaps using such heuristics as availability and representatives (Tversky \& Kahneman, 1974). It follows that, in most applications of decision analysis, decision time may not be a valid index of subjective probability, since probability assessments are often made uniquely. Similarly, in real-life revision of opinion, where the majority of task parameters are nonstationary (Winkler \& Murphy, 1973) and likelihood estimation involves memory search for the previous diagnostic implications of similar data, decision time and subjective uncertainty may be unrelated. Why would one want to use decision time as a measure of subjective probability in decision analysis? The reason is that there is no normative criteria for deciding which assessment method is to be preferred as giving a true, or truer, index of degree of belief. Various direct assessment methods are currently used (for an overview, see Wright, 1984).

Recently, Wright and Ayton (1986) argued that the desirability of an outcome of a predicted event interacts with the direct probability assessed for the event's happening. This desirability effect may have less of an impact on an indirect measure of subjective probability, such as decision time, than on more public pronouncements on the likelihood of events, whick: may be influenced by social context effects. Arguably, Geller and Pitz's (1968) conclusion (cited earlier) that people may be unwilling to reduce their explicit confidence ratings after disconfirming information is an illustration of the effect of social context effects on direct assessments.

Confidence ratings have also been utilized in paired associate learning experiments. For example, Hintzman (1972) demonstrated that the more often responses have 
been given correctly in the past, the more easily subjects discriminate them from errors, shown by higher confidence ratings for correct responses compared to errors. Link (1975), in the context of a study of timed choice discrimination between pairs of sequentially presented similar or different line drawings, found that binary response time serves as a measure of performance that covaries with an extra stimulus factor, the probability of a similar or different pairing. Link (1982) provided a clear discussion of the usefulness of measuring the response times of correct and incorrect responses and detailed a method of correcting response times for guessing. This method requires knowledge of the mean correct response times and the mean incorrect response times.

However, as we have argued, in most applied decision making situations, subjective probability assessments are made uniquely under changing task characteristics under which the correct answer (i.e., whether or not an event in the future will or will not happen) cannot be known; otherwise subjective probability assessment would be unnecessary. It follows that, in this context, the results of these paired associate learning experiments and choice discrimination experiments have limited practical utility.

In the present study, binary decision time increased with subjective task difficulty. The strong linear trend was inconsistent with Hogarth's (1975) model of the nonmonotonic relationship between decision time and task complexity or difficulty. A similar relationship was found between a measure of objective task difficulty and decision time. However, Hogarth's model was originally developed to account for choices between multiattributed alternatives that varied both in number of attributes and in number of attributes shared in common. Perhaps the measures of task difficulty used in the present study of decision making under uncertainty did not adequately capture Hogarth's representation of task complexity. Certainly, Hogarth's model does not have general applicability. Other manipulations of task difficulty are possible with the present design. For example, increasing the number of alternative answers to a question may increase task difficulty by causing response conflict and thus increasing decision time. Similarly, increasing the number of possible probability responses available to a subject may increase probability estimation time. For this reason, written responses to the probability assessment questionnaire (Wright \& Phillips, 1979) may take longer than responses using the restricted number of confidence ranges utilized in the current study.

\section{REFERENCES}

BEACH, L. R., \& PhILLIPS, L. D. (1967). Subjective probabilities inferred from estimates and bets. Journal of Experimental Psychology, 75, 354-359.

Branthwaite, A. (1974). A note comparing three measures of sub- jective probability, their validity and reliability. Acta Psychologica, 38, 337-342.

Cohen, J., Hansel, C. E. M., \& Walker, D. B. (1960). Time taken to decide as a measure of subjective probability. Acta Psychologica, 17, 177-183.

Dalkey, N. (1972). An elementary cross-impact model. Technological Forecasting \& Social Change, 3, 341-351.

DiENER, D., \& ThOMPSON, W. B. (1985). Recognizing randomness. American Journal of Psychology, 98, 433-447.

Ekehammar, B., \& Magnusson, D. (1973). Decision time as a function of subjective confidence and amount of information. Perceptual \& Motor Skills, 36, 329-330.

Fischioff, B., Slovic, P., \& Lichtenstein, S. (1978). Fault-trees: Sensitivity of estimated failure probabilities to problem representation. Journal of Experimental Psychology: Human Perception \& Performance, 4, 330-344.

GAITo, J. (1965). Unequal intervals and unequal $n$ in trend analyses. Psychological Bulletin, 63, 125-127.

Geller, E. S., \& Pitz, G. F. (1968). Confidence and decision speed in the revision of opinion. Organizational Behavior \& Human Performance, 3, 190-201

Glucksberg, S., \& MCCloskey, M. (1981). Decisions about ignorance: Knowing that you don't know, Journal of Experimental Psychology: Human Learning \& Memory, 7, 311-325.

Hintzman, D. L. (1972). Confidence ratings in recall: A re-analysis. Psychological Review, 79, 531-535.

HogarTh, R. M. (1975). Decision time as a function of task complexity. In D. Wendt \& C. A. J. Vlek (Eds.), Utility probability and human decision making (pp. 265-284). Dordrecht: Reidel.

KIRK, R. E. (1982). Experimental design: Procedures for the behavioral sciences (2nd ed.). Belmont, CA: Wadsworth.

LichtensteIN, S., \& Fischioff, B. (1977). Do those who know more also know more about how much they know? Organizational Behavior \& Human Performance, 20, 159-183.

LiNK, S. W. (1975). The relative judgment theory of two choice response time. Journal of Mathematical Psychology, 12, 114-135.

LiNk, S. W. (1982). Correcting response measures for guessing and partial information. Psychological Bulletin, 92, 469-486.

Philuips, L. D., \& WRIGHT, G. (1977). Cultural differences in viewing uncertainty assessing probabilities. In H. Jungerman \& G. de Zeew (Eds.), Decision making and change in human affairs. Amsterdam: Reidel.

RaIfFA, H. (1968). Decision analysis. Reading, MA: Addison-Wesley. SCHEFFE, H. (1953). A method for judging all contrasts in the analysis of variance. Biometrika, $\mathbf{4 0}, 87-104$.

TVersky, A., KAHNEMAN, D. (1974). Judgment under uncertainty: Heuristics and biases. Science, 185, 1124-1131.

WINKLER, R. L. (1967). The assessment of prior distributions in Bayesian analysis. Journal of the American Statistical Association, 62, 776-800.

WINKLER, R. L., \& MURPhy, A. H. (1973). Experiments in the laboratory and the real world. Organizational Behavior \& Human Performance, 10, 252-270.

WrIGHT, G. (1982). Changes in the realism and distribution of probability assessments as a function of question type. Acta Psychologica, 52, 165-174

WRIGHT, G. (1984). Behavioral decision theory. Beverly Hills: Sage. Wright, G., \& AYTON, P. (1986). Subjective confidence in forecasts: A response to Fischhoff and Macgregor. Journal of Forecasting, 5, 117-123.

Wright, G., \& PhILlups, L. D. (1979). Personality and probabilistic thinking. British Journal of Psychology, 70, 295-303.

\section{NOTES}

1. Within-groups degrees of freedom are less than the maximum possible of 252 , because some subjocts did not make use of the whole range of possible responses. 


\section{APPENDIX}

The Probability Assessment Questionnaire (PAQ)

1. Which metal is denser?

(a) Iron

(b) Copper

2. Which contains more proteins per unit weight?

(a) Eggs

(b) Steak (Beef)

3. Did the USSR and Denmark

(a) Join the UN at the same time, or did

(b) The USSR join first?

4. Which has the greater average life expectancy?

(a) Elephant

(b) Man

5. Which is larger?

(a) Black Sea

(b) Caspian Sea

6. Which is the second official language of Finland?

(a) Norwegian

(b) Swedish

7. Aleksandr Solzhenitsyn was awarded the Nobel Prize for Literature for which year?

(a) 1970

(b) 1971

8. How did Henry VIII get rid of Anne of Cleves?

(a) Had her beheaded

(b) Divorced her

9. Buddhism originated in which country?

10. Which is farther north?

(a) China

(b) India

(a) London

(b) New York

(a) Portuguese

(b) Spanish

12. How did Mussolini die?

(a) Suicide

(b) Execution

13. The egg of which bird requires longer incubation?

(a) Chicken

(b) Pigeon

14. In 1963, Valery F. Bykovsky orbited the earth in Vostok V. Was this cosmonaut

(a) A man, or

(b) A woman

15. Which planet takes longer to go round the sun once?

(a) Earth

(b) Mars

16. Which does Japan produce more of (by weight)?

(a) Wheat

(b) Rice

17. Which was formed first?

(a) South-East Asia Treaty Organization

(b) North Atlantic Treaty Organization

18. Biennial means

(a) Twice a year

(b) Once every two years

19. Which animal does not eat meat?

(a) Badger

(b) Hare

20. Lacrosse was developed from a game played by what people?

(a) American Indians

(b) Asian Indians

21. Where are the Atlas Mountains?

(a) Europe

(b) Africa

22. Which country has the larger land area?

(a) USSR

(b) China

23. Which is larger in terms of surface area?

(a) Atlantic Ocean

(b) Pacific Ocean

24. Which type of vessels carry blood from the heart?

(a) Artery

(b) Vein 


\section{APPENDIX (Continued)}

25. Which civilization came first?

26. Which is nearer London?

27. Who "discovered" New Zealand?

28. When did the People's Republic of China join the UN?

29. Which legs do cows use first when getting up from the ground?

30. Which is the smallest country in South America?

31. Where was Frank Sinatra born?

32. Which is longer?

33. Which was "discovered" first?

34. Which tropic is north of the Equator?

35. When were the modern Olympic Games started?

36. There are more calories in one cup of which vegetable?

37. Which camel has two humps?

38. What is the capital of New Zealand?

39. Which country has the greater number of ships on its register?

40. Which country produces more natural nubber?

41. The balalaika is a musical instrument from which country?

42. Which language is spoken by the greater number of people as their mother tongue?

43. Which is farther south?

44. Which capital city has the larger population?

45. Who was born earlier?

46. In the Book of Genesis, did

47. What is a young eel called?

48. Who carried the world on his shoulders?

49. Are fishes

50. Which country has the larger population? (a) Greek

(b) Roman

(a) New York

(b) Moscow

(a) Abel Tasman

(b) Captain Cook

(a) 1972

(b) 1971

(a) Hind legs

(b) Front legs

(a) Ecuador

(b) Uruguay

(a) Sicily

(b) USA

(a) Panama Canal

(b) Suez Canal

(a) Einstein's Theory of Relativity

(b) Aspirin

(a) Capricorn

(b) Cancer

(a) 19th Century

(b) 20th Century

(a) Fresh corn cut from the cob

(b) Shelled green peas

(a) Bactrian

(b) Arabian

(a) Auckland

(b) Wellington

(a) Liberia

(b) Panama

(a) Brazil

(b) Malaya

(a) Russia

(b) Greece

(a) English

(b) Chinese

(a) Cape of Good Hope

(b) Cape Horn

(a) Peking

(b) Tokyo

(a) Alexander the Great

(b) Jesus Christ

(a) Abel kill Cain, or

(b) Cain kill Abel?

(a) Leveret

(b) Elver

(a) Hercules

(b) Atlas

(a) Cold-blooded, or

(b) Warm blooded

(a) India

(b) China 
APPENDIX (Continued)

51. Who is the President of South Africa (1974)?

(a) B. J. Vorster

(b) J. J. Fouche

52. What is a flying fox?

(a) A tree-squirrel

(b) A bat

53. Which river is longer?

(a) Nile

(b) Amazon

54. The Xerox copying process was discovered

(a) Before World War II

(b) After World War II

55. Which country suffered more battle deaths in World War II?

(a) Germany (including Austria)

(b) Japan

56. In boxing, which is lighter?

(a) Featherweight

(b) Flyweight

57. What nationality was the painter Rubens?

(a) Dutch

(b) Flemish

58. Who wrote Wuthering Heights?

(a) Emily Brontöe

(b) Charlotte Brontöe

59. Which has more Vitamin $C$ per ounce?

(a) Lemon juice

(b) Grapefruit juice

60. Which is larger?

(a) Horse

(b) Moose

61. What is a bryologist?

(a) Expert on eggs

(b) Expert on mosses

62. Which is farther east?

(a) Moscow

(b) Cape Town

63. In which country was the Titanic registered?

(a) USA

(b) Britain

64. Which is the larger country in terms of area?

(a) France

(b) Spain

65. Which planet is nearer the sun?

(a) Mars

(b) Venus

66. For which is the average gestation period longer?

(a) Man

(b) Chimpanzee

67. The month has more days on which calendar?

(a) Lunar

(b) Solar

68. What is the modern name for Persia?

(a) Iraq

(b) Iran

69. Which country is larger in terms of area?

(a) Liechtenstein

(b) Luxembourg

70. Tin cans contain more of which metal?

(a) Iron

(b) Tin

71. What is the capital of Tasmania?

(a) Launceston

(b) Hobart

72. Which part of the Bible has more books?

(a) Old Testament

(b) New Testament

73. Which continent is Damascus in?

(a) Africa

(b) Asia

74. Where was Shakespeare born?

(a) Stratford-on-Avon

(b) London

75. Acids turn litmus paper to what color?

(a) Red

(b) Blue 